\title{
Elucidating the Thermodynamic Driving Forces of Polyanion- Templated Virus-like Particle Assembly
}

\author{
Stan J. Maassen, ${ }^{\dagger}$ Jurriaan Huskens, $^{\ddagger 0}$ and Jeroen J. L. M. Cornelissen ${ }^{*}{ }^{\dagger}$ (1) \\ ${ }^{\dagger}$ Laboratory of Biomolecular Nanotechnology, MESA+ Institute for Nanotechnology and ${ }^{\ddagger}$ Molecular NanoFabrication Group, \\ MESA+ Institute for Nanotechnology, University of Twente, Enschede 7500 AE, The Netherlands
}

\section{Supporting Information}

\begin{abstract}
A virus in its most simple form is comprised of a protein capsid that surrounds and protects the viral genome. The self-assembly of such structures, however, is a highly complex, multiprotein, multiinteraction process and has been a topic of study for a number of years. This selfassembly process is driven by the (mainly electrostatic) interaction between the capsid proteins (CPs) and the genome as well as by the protein-protein interactions, which primarily rely on hydrophobic interactions. Insight in the thermodynamics that is involved in virus and virus-like particle (VLP) formation is crucial in the detailed understanding of this complex assembly process. Therefore, we studied the assembly of CPs of the cowpea chlorotic mottle virus (CCMV) templated by polyanionic species (cargo), that is, single-stranded DNA (ssDNA), and polystyrene sulfonate (PSS) using isothermal titration calorimetry. By separating the electrostatic $\mathrm{CP}$-cargo

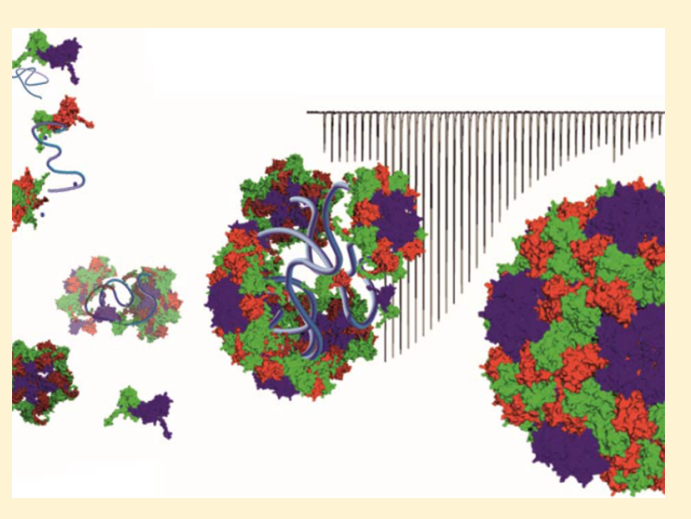
interaction from the full assembly interaction, we conclude that $\mathrm{CP}-\mathrm{CP}$ interactions cause an enthalpy change of -3 to $-4 \mathrm{kcal} \mathrm{mol}^{-1} \mathrm{CP}$. Furthermore, we quantify that upon reducing the $\mathrm{CP}-\mathrm{CP}$ interaction, in the case of CCMV by increasing the $\mathrm{pH}$ to 7 , the $\mathrm{CP}$-cargo starts to dominate VLP formation. This is highlighted by the three times higher affinity between CP and PSS compared to CP and ssDNA, resulting in the disassembly of CCMV at neutral $\mathrm{pH}$ in the presence of PSS to yield PSS-filled VLPs.
\end{abstract}

\section{INTRODUCTION}

By combining proteins and synthetic materials, such as polymers or nanoparticles, new materials have been developed with unique properties. ${ }^{1-3}$ In this respect, a specific group of proteins, those derived from viruses, has gained interest due to the ability of viral proteins to form highly symmetrical and homogenous structures. ${ }^{4-8}$ Research in this direction has already shown the use of viruses and their components in a wide range of fields with applications, for example, in electronics and energy storage, ${ }^{9,10}$ in drug delivery and medicine, ${ }^{11-14}$ and as catalytic particles. ${ }^{15-18}$

To advance in these fields, we need to understand the interactions taking place between the various components of such materials. A lot of studies focus on the self-assembly of virus proteins into empty spheres often triggered by altering the solution environment of the proteins. ${ }^{19-24}$ For example, in the case of the cowpea chlorotic mottle virus (CCMV), the self-assembly of capsid proteins (CPs) can be triggered by changing the $\mathrm{pH}^{19,20}$ However, under native conditions, that is, involving the wild-type virus, as well as for many applications, capsids are generally filled with a cargo. ${ }^{25-28}$ To fully understand the interactions between the structure subunits, also, CP-cargo interactions should be studied. Previous research in this direction has already shown that the cargo can aid in virus-like particle (VLP) formation, specifically in the early stages of assembly. ${ }^{22,29}$ Association of
$\mathrm{CP}$ with cargo, often involving electrostatic interactions, places CPs in close proximity. This close contact between the proteins allows for maturation of the unstructured $\mathrm{CP}-$ cargo aggregates into virus-like particles. ${ }^{29}$

Here, we describe a study on a virus(-like) particle assembly and the thermodynamic contributions involved. To this end, we have employed isothermal titration calorimetry (ITC) to monitor heat changes caused by the interaction between $\mathrm{CP}$ and cargo upon mixing. ITC has been used in viral research before, for example, to study membrane fusion events for the influenza virus, ${ }^{30}$ DNA ejection from bacteriophages, ${ }^{31}$ and the binding of inhibitors to viruses for the development of new antiviral treatments. ${ }^{32,33}$ Moreover, ITC has been applied by De Souza et al. to qualitatively study the assembly of hepatitis $\mathrm{C}$ virus core proteins around a nucleic acid. ${ }^{34}$ Although they did not quantify the binding or assembly energies, they suggested that the assembly is mainly driven by electrostatic interactions between the protein and the nucleic acid. In contrast to this work, we attempt to understand the $\mathrm{CP}$-cargo interactions not only qualitatively but also quantitatively, to gain understanding on the cargo-related aspects that stabilize or destabilize viruses and VLPs. Insights that are not only

Received: July 1, 2019

Revised: October 1, 2019

Published: October 29, 2019 
relevant for the design of new materials but can also help in developing new antiviral drugs that act by the removal of the genetic materials from the virus capsid.

For this purpose, we studied the assembly of isolated CCMV CPs around two types of polyanionic cargos, single-stranded DNA (ssDNA) and polystyrene sulfonate (PSS). Besides using ITC to study the enthalpic contributions, we applied analytical techniques, such as dynamic light scattering (DLS), sizeexclusion chromatography (SEC), and electron microscopy (EM) as well as microscale thermophoresis (MST) to further study the assembly processes and for structural studies.

\section{RESULTS AND DISCUSSION}

Our initial experiments focused on the stability of CCMV and how well its capsids retain their cargo. The stability of the virus particles indicates how strongly the subunits interact under the used conditions and how easily the particles release their cargo. For viruses as well as for the use of VLPs in, for example, drug delivery, stability is crucial during transportation to the host or target cells, while the release of the cargo is needed for infection or efficacy. Furthermore, the speed of assembly and disassembly of particles may give insight into the mechanisms involved and the pathways that are followed. ${ }^{25,29,35}$ To study these aspects, we mixed wild-type CCMV with PSS at $\mathrm{pH} 5$ where $\mathrm{CP}-\mathrm{CP}$ interactions are strong, and at $\mathrm{pH} 7.5$ where $\mathrm{CP}-\mathrm{CP}$ interactions are reduced due to deprotonation of carboxylic acid groups on the proteins, ${ }^{36,37}$ and the particles rely on $\mathrm{CP}$-cargo interactions for stability (see Figures S1S2). ${ }^{38}$ At $\mathrm{pH} 5$, we observed that the CCMV particles remain stable upon mixing (see Figure $\mathrm{S} 1 \mathrm{a}, \mathrm{c}$ ). However, at $\mathrm{pH}$ 7.5, we observed disassembly of the CCMV particles and the formation of PSS-filled VLPs (see Figures S1b,d and S2), as witnessed by the appearance of a peak at an elution volume of $V \sim 12 \mathrm{~mL}$ in SEC, indicating the presence of smaller particles. In Figure S1a,b, the absorbance at $\lambda=548 \mathrm{~nm}$, originating from a rhodamine dye on the PSS $\left(\lambda_{\max }=548 \mathrm{~nm}\right)$, indicates that this fraction indeed contains PSS. Furthermore, sodium dodecyl sulfate-polyacrylamide gel electrophoresis (SDSPAGE) was applied to confirm the presence of CP in this fraction, while transmission electron microscopy (TEM) was used to visualize the particles formed at $\mathrm{pH} 7.5$ (Figure S2). These results underline that the exchange of the cargo is only possibly when the cage interactions are sufficiently weakened to provide dynamic exchange of cage constituents. When the cage interactions are too strong, the structures are kinetically trapped, preventing reorganization into a thermodynamically more favored structure.

\section{PSS-TEMPLATED ASSEMBLY}

In an attempt to explain the formation of PSS-filled VLPs when mixing CCMV with PSS at $\mathrm{pH} 7.5$, we studied the interactions between CCMV CP and polyanionic cargos. We started by studying the VLP assembly templated by PSS. To do this, the $\mathrm{CP}$ of CCMV was isolated by disassembling the virus and removing the viral ssRNA. Subsequently, we performed DLS and MST measurements at various CP-PSS ratios to gain insight in PSS-templated VLP formation (Figure 1). In this way, the disassembly step of the exchange process, which is assumed to be slow due to kinetic stabilization, ${ }^{39}$ described above can be circumvented, and the, most likely fast, reassembly step can be investigated separately and possibly at thermodynamic equilibrium.
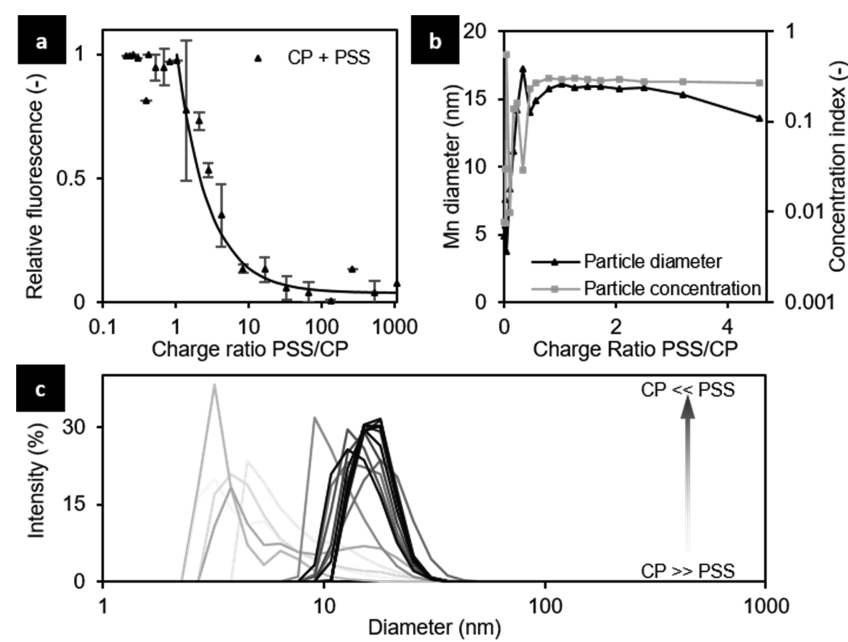

Figure 1. (a) MST measurements at various PSS:CP ratios (black triangles; expressed as charge ratio, assuming one negative charge per PSS monomer unit and 10 positive charges per $\mathrm{CP}$ ) and a trendline showing the calculated fluorescence for various ratios of encapsulated to free PSS based on a relative fluorescence of 1 for fully encapsulated PSS and a relative fluorescence $=0.04$ for PSS (average of the last 4 data points) (black line) and assuming full particle formation. (b) DLS data at various PSS:CP ratios, showing particle size (black triangles) and concentrations (gray squares). The black and gray lines are guides to the eye. (c) Number average DLS data of samples going from excess CP (light gray) to excess PSS (black) at a constant CP concentration.

The MST data shown in Figure la were obtained by following the thermophoresis of rhodamine-labeled PSS at varying concentrations of CP. From low charge ratios of PSS to $\mathrm{CP}$, that is, at excess $\mathrm{CP}$, to a charge ratio of $\sim 1$, very similar fluorescence responses were measured. Upon increasing the charge ratio above 1 , we observed a decrease in the fluorescence response, which leveled off to close to zero at very high charge ratios, that is, at excess PSS. We have added a trendline that plots the fluorescence intensity expected when quantitative encapsulation of PSS occurs, while assuming that the interaction stoichiometry is optimal at a charge-neutral ratio. Because of the fit of this trendline with the experimentally observed data points, we assume that the decrease in fluorescence intensity is merely an effect of having an increased concentration of free PSS in the solution at higher charge ratios rather than having different amounts of $\mathrm{CP}$ binding to PSS at different charge ratios. Based on that interpretation, we conclude that, at charge ratios $<1$, we have solutions of fully bound PSS, attributed to PSS-filled VLPs, and free $\mathrm{CP}$, while at charge ratios $>1$, we have solutions containing PSS-filled VLPs and free PSS. Thus, the assembly is practically fully formed at all charge ratios leaving the concentration of the unbound fraction of the minor component (CP or PSS) too low to measure, therefore precluding determination of the binding constant from these data.

These conclusions are supported by the DLS data shown in Figure $1 b, c$ where we titrated PSS into a solution containing CP. At low charge ratios, the number average diameter steadily increased up to $M_{\mathrm{n}} \sim 16.5 \mathrm{~nm}$ at a charge ratio of $\sim 1$ after which it became stable. The same trend was observed in the concentration index, a unitless indication of the particle concentration in a DLS sample as determined by the instrument. This indicates that, above a charge ratio of 1 , the concentration of VLPs was constant. Figure $1 b, c$ shows 
increasing peak at $M_{\mathrm{n}} \sim 17 \mathrm{~nm}$ when going from samples with excess CP (light gray) to samples with an excess of PSS (black).

Next, we applied ITC to observe the heat changes occurring upon mixing of the components, in an attempt to get quantitative information on the thermodynamics and/or interaction enthalpies that are associated with their selfassembly. The assembly of VLPs is highly complex, involving many components in various stoichiometries and therefore involving many interactions in a variety of stoichiometries. Yet, the process is presumed to be highly cooperative, leading to the absence of measurable amounts of intermediates and allowing visualization of the start and end situations of the selfassembly process only. The MST data shown above indicate that the assembly formation may be quantitative at any experimentally accessible concentration so that determination of an equilibrium constant may be impossible. Yet, valuable stoichiometry and enthalpy information should be attainable.

To allow the interpretation of the thermodynamic driving forces, we assume that the enthalpy change $(\Delta H)$ of the system consists of two contributions: the enthalpy change caused by the electrostatic interaction between the CP Nterminus and the PSS $\left(\Delta H_{\mathrm{CP}-\mathrm{PSS}}\right)$, and the enthalpy change caused by the interaction between different $\mathrm{CP}$ subunits leading to VLP formation $\left(\Delta H_{\mathrm{CP}-\mathrm{CP}}\right)$. To determine $\Delta H_{\mathrm{CP}-\mathrm{PSS}}$, we performed ITC measurements involving the titration of PSS into a solution of a peptide consisting of 26 amino acids corresponding to CCMV CP's N-terminal 26 amino acids (N-term). This $\mathrm{N}$-terminal section of the $\mathrm{CP}$ contains the ten positive charges that interact with negative cargos. In this way, we measure the electrostatic interactions without involving the $\mathrm{CP}-\mathrm{CP}$ interactions. In this approach, we assume that no allosteric binding behavior occurs, meaning that $\Delta H_{\mathrm{CP}-\mathrm{PSS}}$ is not affected by the fact that CCMV CP is present in a dimeric form or by the VLP assembly process. Small conformational changes are expected for capsid assembly according to the quasi-equivalence theory; ${ }^{40}$ however, as these occur after the initial $\mathrm{CP}$-cargo interaction, these affect $\Delta H_{\mathrm{CP}-\mathrm{CP}}$ rather than $\Delta H_{\mathrm{CP}-\mathrm{PSS}}$. Figure 2 shows representative data of titrations of PSS into N-term solutions and compares them to data from similar experiments with $\mathrm{CP}$ instead of $\mathrm{N}$ term. In these measurements, we titrated two lengths of PSS, with $\mathrm{Mw} \sim 6.7$ and $\sim 70 \mathrm{kDa}$, into solutions of either $\mathrm{N}$-term or $\mathrm{CP}$ while monitoring the heat supplied by the heater coil and compared the heat effects that occur. In these experiments, the concentration of CP was kept constant $(0.075 \mathrm{mM})$. For both 6.7 and $70 \mathrm{kDa}$ PSS, the same mass per volume ratio was used, thus keeping the number of monomer units added per injection constant. This automatically means that, due to the difference in the chain length, the number of mole PSS added per injection is not equal between the two types of PSS, but the obtained charge ratios are directly comparable. To prevent any effects of differences between batches of CP, for example, protein purity, we present and compare samples that were prepared from the same batch of protein. Figure 2a,b presents the same data as Figure $2 \mathrm{c}, \mathrm{d}$; however, to be able to compare the different samples, in Figure 2a,b, the data are normalized to heat per molecular fragment of 10 charges; whereas in Figure $2 c, d$, the data are normalized to heat per mole of PSS.

We assume that the $\mathrm{CP} \mathrm{N}$-termini, having ten positive charges, ${ }^{41}$ interact with a segment of ten negative charges on the PSS, leading to charge compensation. This is in line with the DLS and MST measurements discussed earlier where
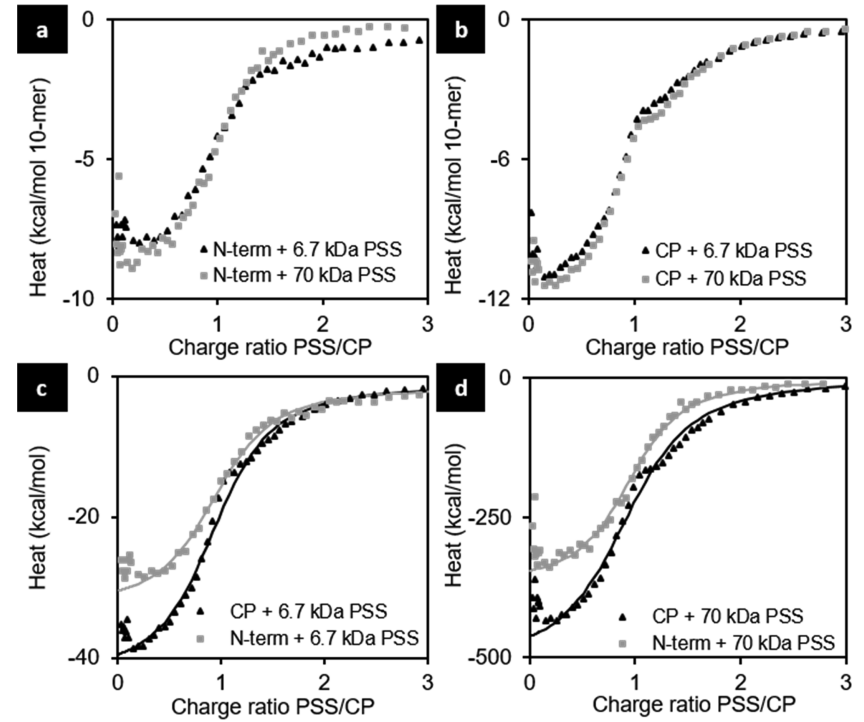

Figure 2. Heat changes per injection, normalized to heat per mole of a molecular fragment containing ten charges, of $6.7 \mathrm{kDa}$ (black) and $70 \mathrm{kDa}$ PSS (gray), into a solution of (a) N-term or (b) CP, as a function of the charge ratio. Heat changes per injection, in heat per mole PSS, for titrations of (c) $6.7 \mathrm{kDa}$ PSS and (d) $70 \mathrm{kDa}$ PSS into $\mathrm{N}$-term (gray) or CP (black) solutions. The line fits presented in (c) and (d) are based on a single-binding site model. In these experiments, PSS in $\mathrm{pH} 7.5$ buffer $\left(2 \mathrm{mg} \mathrm{mL}^{-1}\right.$ PSS in $50 \mathrm{mM}$ Tris- $\mathrm{HCl}, 0.3 \mathrm{M} \mathrm{NaCl}$ ) is titrated into solutions of $\mathrm{CP}$ or $\mathrm{N}$-term $(0.075 \mathrm{mM})$ in the same buffer.

particle formation occurs until a charge ratio of PSS to CP of $\sim 1$ is reached. When we correct the heat changes in ITC for the amount of charges added, which was kept constant between the different experiments, by calculating the heat per mole of segments of ten negative charges (10-mer) added, we see that for both the interactions of PSS with N-term as well as that of PSS with CP, the difference in the PSS length does not affect the thermodynamics of the system, as witnessed by similar curves in Figure 2a,b for 6.7 and $70 \mathrm{kDa}$ PSS. Indeed, when looking at the heat per mole of PSS, without correcting for the number of charges per PSS chain, significant differences in heat between titrations with $6.7 \mathrm{kDa}$ PSS (Figure 2c) and 70 $\mathrm{kDa}$ PSS (Figure 2d) are measured, corresponding to the difference in lengths. When comparing the data involving the $\mathrm{N}$-term with the data involving $\mathrm{CP}$, we observe an increase in the heat released when adding PSS to CP compared to adding PSS to N-term (Figure 2c,d). Furthermore, the ITC curves of adding PSS to a solution of CP show a kink at a charge ratio of approximately 1 , which is not observed in the curves involving $\mathrm{N}$-term.

The ITC data for titrations of PSS in solutions of N-term were fitted with a simple 1:1 thermodynamic model (Figure $2 \mathrm{c}, \mathrm{d})$, gray solid lines). This model is based on the set of equilibrium and mass balance equations given below where all species are assumed to be 10 -mers.

$$
\begin{aligned}
& K=\frac{[\mathrm{N} \text {-term }-\mathrm{PSS}]}{[\mathrm{N} \text {-term }] \times[\mathrm{PSS}]} \\
& \mathrm{N} \text {-term }{ }_{\text {tot }}=[\mathrm{N} \text {-term }]+[\mathrm{N} \text {-term }-\mathrm{PSS}] \\
& \mathrm{PSS}_{\text {tot }}=[\mathrm{PSS}]+[\mathrm{N} \text {-term }-\mathrm{PSS}]
\end{aligned}
$$


Table 1. Summary of Thermodynamic Parameters Obtained by Fitting ITC Data (per Mole of 10-Mer) to a 1:1 Binding Model $^{42}$

\begin{tabular}{lccc}
\multicolumn{1}{c}{ sample } & $K\left(\times 10^{5} \mathrm{M}^{-1}\right)$ & $\Delta H\left(\mathrm{kcal} \mathrm{mol}^{-1}\right)$ & $T \Delta\left(\mathrm{kcal} \mathrm{mol}^{-1}\right)$ \\
$\mathrm{N}$-term + 6.7 kDa PSS & 2 & -8.9 & -1.7 \\
$\mathrm{~N}$-term + 70 kDa PSS & 2 & -9.5 & -2.2 \\
$\mathrm{CP}+6.7 \mathrm{kDa}$ PSS & 1.5 & -11.9 & -4.8 \\
$\mathrm{CP}+70 \mathrm{kDa}$ PSS & 1.5 & -13.0 & -6.0 \\
$\mathrm{~N}-$ term $+6.7 \mathrm{kDa}$ PSS 0.6 M NaCl & 0.4 & -8.6 & -2.2 \\
$\mathrm{CP}+6.7 \mathrm{kDa}$ PSS 0.6 M NaCl & 1.5 & -9.5 & -2.4 \\
$\mathrm{CP}+6.7 \mathrm{kDa}$ PSS (batch 2) & 2.7 & -11.2 & -3.8 \\
$\mathrm{CP}+$ ssDA & 1.0 & -1.8 & 5.0
\end{tabular}

Here, $K$ is the equilibrium constant, $\mathrm{N}$-term tot $_{\text {and }}$ asS tot $_{\text {are }}$ the total concentrations of $\mathrm{N}$-term and PSS in the solution, respectively, $[\mathrm{N}$-term] is the concentration of free $\mathrm{N}$-term, [PSS] is the concentration of free 10-meric PSS, and [Nterm-PSS] is the concentration of the N-term-PSS complex. The model was used to determine $K$, which gives the concentrations of the various components, and $\Delta H$, which gives the amount of heat produced per mole of the (10-meric) $\mathrm{N}$-term-PSS complex formed.

The model assuming 1:1 complexation of 10-mers fits our data well, which suggests that one $\mathrm{N}$-term interacts with a PSS 10-mer chain segment, leading to charge compensation. When viewing realistic PSS lengths, all N-term-PSS segment interactions occur according to an independent binding site model. Since mathematically, an independent binding site model with a PSS:N-term stoichiometry of $1: 3.6$ (for $6.7 \mathrm{kDa}$ PSS) is equal to an independent binding site model with a stoichiometry of 1:1 with 3.6 times higher concentration of PSS with a length of 10 monomers; for data fitting, a concentration of 10-mers of PSS was used.

Using this thermodynamic model, we obtained values for $K$ and $\Delta H$ for the $\mathrm{N}$-term-PSS interactions. We assume that these thermodynamic parameters also correspond to the electrostatic interaction of CP-PSS while excluding VLP assembly (a full overview of thermodynamic parameters determined here is given in Table 1).

For both 6.7 and $70 \mathrm{kDa}$ PSS interacting with $\mathrm{N}$-term, $\mathrm{K}$ values of $\sim 2 \times 10^{5} \mathrm{M}^{-1}$ were found. The values for $\Delta H$, which we assume to be equal to $\Delta H_{\mathrm{CP}-\mathrm{PSS}}$, were -8.9 and $-9.5 \mathrm{kcal}$ $\mathrm{mol}^{-1}$ for 6.7 and $70 \mathrm{kDa}$ PSS, respectively. From these data, we calculated $T \Delta S$ values of -1.7 and $-2.2 \mathrm{kcal} \mathrm{mol}^{-1}$ for 6.7 and $70 \mathrm{kDa}$ PSS, respectively. These values indicate that the assembly processes are exothermic and mainly enthalpy driven, with a negative entropic contribution. This negative contribution could be due to the fixation of conformations upon assembly. The negative entropic effect, however, is only small, which may be due to the release of counter ions that contribute positively to the entropy of the system.

Knowing the contribution of the electrostatic interactions between CP and PSS, we analyzed the ITC measurements involving PSS titrations into CP solutions using a similar approach. Looking more closely into the ITC data for the interaction of CP with 6.7 and $70 \mathrm{kDa}$ PSS, we observe a bend in both curves at a charge ratio of $\sim 1$ (see Figure $2 b$ ). This bend suggests that multiple interactions may be involved in this system as well as complexes or intermediates with different stoichiometries. This is not surprising, considering the complexity of VLP assembly and the large number of components in forming such particles. The location of this bend suggests that this second binding event has a PSS:CP stoichiometry higher than $1: 1$. In an attempt to determine the thermodynamic parameters accurately, we tried to model two different binding events: first, the electrostatic binding of CP to PSS (i.e., CP-PSS interactions), and second, the rearrangement of CPs to higher-order structures (CP-CP interactions). Unfortunately, we were unable to develop a model that could accurately fit the data fully. For this reason and because of the otherwise large similarities between the data for $\mathrm{N}$-term and $\mathrm{CP}$, we applied the binding model described earlier for the $\mathrm{N}$ term-PSS system on the CP-PSS systems as well to get a rough estimate of the thermodynamic parameters (Table 1 ). By assuming that the changes in heat are caused by two events, the electrostatic interactions of the $\mathrm{CP} \mathrm{N}$-terminus with the PSS and the assembly of the CP-PSS complexes into VLPs due to $\mathrm{CP}-\mathrm{CP}$ interactions, we can obtain information on the $\mathrm{CP}-\mathrm{CP}$ interactions by comparing the thermodynamic parameters of $\mathrm{N}$-term-PSS with the thermodynamics of the full assembly.

For titration of PSS into CP solutions, $K$ values of $\sim 1.5 \times$ $10^{5} \mathrm{M}^{-1}$ were found, while $\Delta H$ values were -11.9 and -13.0 $\mathrm{kcal} \mathrm{mol}^{-1}$ for 6.7 and $70 \mathrm{kDa}$ PSS, respectively (see Table 1 ). It is important to note, also underlined by the quantitative assembly formation observed by MST as described above, that fully assembled VLPs are assumed to be formed quantitatively also under the ITC conditions. This means that the gradual curve in the heat changes, which provides the apparent values of $K$, probably reflect gradual affinity and heat changes during the progress of the titration and/or contributions from cooperative and/or multivalent behavior. The lack of proper models with such high stoichiometries and the absence of experimental observations of intermediates preclude the development of better models at this stage. Assuming $\Delta H$ in these measurements consist of $\Delta H_{\mathrm{CP}-\mathrm{PSS}}$ and $\Delta H_{\mathrm{CP}-\mathrm{CP}}$, we can calculate $\Delta H_{\mathrm{CP}-\mathrm{CP}}$, giving -3.0 and $-3.5 \mathrm{kcal} \mathrm{mol}^{-1}$ for the titrations of 6.7 and $70 \mathrm{kDa}$ PSS into $\mathrm{CP}$ solutions, respectively. Since $\Delta H_{\mathrm{CP}-\mathrm{CP}}$ involves $\mathrm{CP}-\mathrm{CP}$ interactions rather than $\mathrm{CP}-\mathrm{PSS}$ interactions, similar values for $\Delta H_{\mathrm{CP}-\mathrm{CP}}$ were expected independent of the length of PSS, assuming particles of the same size are formed (as discussed below). Indeed, the values calculated for $\Delta H_{\mathrm{CP}-\mathrm{CP}}$ at 6.7 and $70 \mathrm{kDa}$ PSS are comparable, giving an estimated (exothermic) heat change of approximately -3 to $-4 \mathrm{kcal} \mathrm{mol}^{-1}$. Interestingly, values of the same order of magnitude were found to regulate CCMV CP self-assembly at acidic $\mathrm{pH}$, having a $\mathrm{K} \sim 2.5 \times 10^{5}$ $\mathrm{M}^{-1}$ and association energies of $\sim-3.5 \mathrm{kcal} \mathrm{mol}^{-1}$. ${ }^{43}$

The changes in entropy, $T \Delta S$, for these systems were calculated to be -4.8 and $-6.0 \mathrm{kcal} \mathrm{mol}^{-1}$ for 6.7 and $70 \mathrm{kDa}$ PSS, respectively. Comparing these values to the entropy changes for N-term PSS interactions, we determine that, upon VLP assembly, the system loses entropy, approximately -3 to 
$-4 \mathrm{kcal} \mathrm{mol}^{-1}$ difference is calculated between $T \Delta S$ of the $\mathrm{N}$ term-PSS interactions and TAS of the CP-PSS interactions. According to literature, $\mathrm{CP}-\mathrm{CP}$ interactions are driven by hydrophobic interactions, ${ }^{44,45}$ which are commonly accompanied by favorable entropies and unfavorable enthalpy changes. We expected that burying of hydrophobic patches on the CPs and the release of water molecules from these regions that drive the $\mathrm{CP}-\mathrm{CP}$ interactions would cause a gain in entropy and would therefore aid VLP assembly. However, our data suggest that this effect is less than other, entropically unfavorable, events, such as possibly the restriction of CP and PSS mobility upon assembly and cage formation, where many free components assemble into a single structure thus reducing the freedom of the subunits. It should be noted that the CP$\mathrm{CP}$ interactions are $\mathrm{pH}$ dependent and that at elevated $\mathrm{pH}$ (>6.5) CP-CP interactions become weak and have different molecular origin due to deprotonation of carboxylic acid groups, which means different thermodynamic effects may be observed at different $\mathrm{pH}$. Moreover, reducing the mobility of ionic species in the samples possibly also reduces the systems entropy. ${ }^{46}$

We extended this study by looking at the effect of ionic strength on the thermodynamics of this system by performing titrations at $0.6 \mathrm{M} \mathrm{NaCl}$, in contrast to $0.3 \mathrm{M}$ in previous experiments (Figure 3). From Figure 3a, we observe that at 0.6
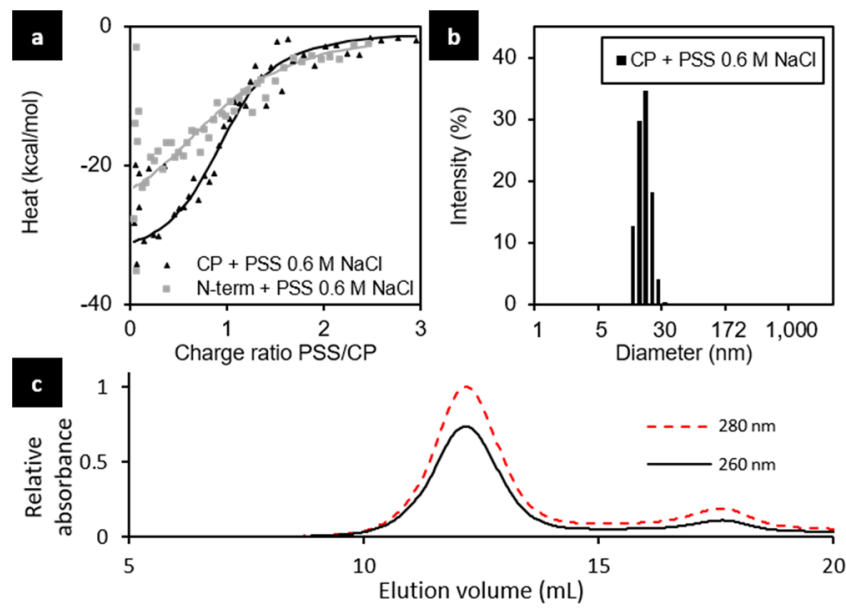

Figure 3. (a) Integrated data of the measured temperature change per injection of $6.7 \mathrm{kDa}$ PSS into a solution of CP (black triangles) or Nterm (gray squares). The black and gray lines show the fitted data based for the CP and N-term, respectively. (b) DLS and (c) SEC measurements of the product after ITC.

$\mathrm{M} \mathrm{NaCl}$ the interactions between PSS and CP or N-term gave lower changes in heat compared to the $0.3 \mathrm{M} \mathrm{NaCl}$ samples. However, upon data fitting of the data for PSS titrated to an Nterm solution, we found that mainly $K$ is affected, giving a value of $4.4 \times 10^{4} \mathrm{M}^{-1}$, which is an order of magnitude smaller than at lower ionic strength. The $\Delta H$ of the N-term-PSS interaction, which yields $\Delta H_{\mathrm{CP}-\mathrm{PSS}}$, was found to be -8.6 $\mathrm{kcal} \mathrm{mol}^{-1}$, which is comparable to the values found at lower ionic strength. On the other hand, when looking at the data for the titration of PSS into a CP solution, we found $K$ to be $1.5 \times$ $10^{5} \mathrm{M}^{-1}$, which is in the same range as similar measurements done at $0.3 \mathrm{M} \mathrm{NaCl} . \Delta H$ for this titration was found to be $-9.5 \mathrm{kcal} \mathrm{mol}^{-1}$, giving a $\Delta H_{\mathrm{CP}-\mathrm{CP}}$ of $-0.9 \mathrm{kcal} \mathrm{mol}^{-1}$, which is lower than values found at lower ionic strength. We calculated $T \Delta S$ values of -4.8 and $\sim-2.4 \mathrm{kcal} \mathrm{mol}^{-1}$ for PSS titrations into $\mathrm{CP}$ solutions at 0.3 and $0.6 \mathrm{M} \mathrm{NaCl}$, respectively. As mentioned, $\mathrm{CP}-\mathrm{CP}$ interactions are of a hydrophobic nature, which mainly gives a favorable entropy rather than enthalpy change. Increasing the ionic strength also enhances hydrophobic effects and may therefore increase the change in entropy. ${ }^{47}$ The calculated values indeed indicate more favorable entropic effects at higher ionic strength.

When comparing all data for $6.7 \mathrm{kDa}$ PSS with that of 70 $\mathrm{kDa}$ PSS, both lengths of PSS show very similar thermodynamic behavior. Furthermore, both 6.7 and $70 \mathrm{kDa}$ PSS induce $T=1$ particle formation, which is comprised of $60 \mathrm{CPs}$ and consequently 600 positive charges. This means that, upon charge compensation, approximately 17 chains of $6.7 \mathrm{kDa}$ PSS $(\sim 36$ negative charges each) and 2 chains of $70 \mathrm{kDa}$ PSS ( $\sim 380$ negative charges each) are encapsulated. Higher $T$ numbers might accessible by with even longer PSS, which may also lead to a different charge ratio per VLP. This may cause an excess of negative charges, as occurs naturally in the native virus where $\sim 3000 \mathrm{nt}$ (3000 negative charges) ssRNA is encapsulated in a $T=3(180 \mathrm{CPs}=1800$ positive charges $){ }^{48}$ Also, encapsulating high molecular weight PSS as described by $\mathrm{Hu}$ et al. ${ }^{49}$ yielded negative overcharging, while an excess of positive charges is observed if particles with a higher $T$ number are formed, as shown by Cadena-Nava et al. when measuring the number of PSS chains per capsid. ${ }^{50}$ These variations clearly show that the encapsulation of polyanions is dependent on the assembly conditions used and does not merely depend on the number of charges. We expect that significantly increasing the length of PSS or changing the assembly conditions may affect the thermodynamics of the system. However, according to the data shown in Figure 2, the lengths of PSS used here show similar thermodynamic behavior.

Comparison of CP-PSS and CP-ssDNA Interactions. To further study the CP-cargo interactions, we applied ITC to compare PSS- or ssDNA-templated VLP assembly. PSS (6.7 and $70 \mathrm{kDa}$ ) or ssDNA (obtained from salmon testes, $\sim 500-$ $800 \mathrm{nt}$, on average 700 negative charges) was titrated into a CP solution under identical conditions while monitoring the heat supplied by the heater coil. Since the $\mathrm{CP}$-cargo interactions are of an electrostatic nature, the concentration of $\mathrm{CP}$ and the concentration of negative charge added were kept constant $(\sim 1 \mathrm{mg} / \mathrm{mL})$ during these experiments, to be able to compare the interactions. Furthermore, samples that are compared were prepared from the same batch of $\mathrm{CP}$, to exclude effects of differences between different batches, for example, protein purity. Representative integrated ITC data of such measurements are shown in Figure 4.

The assembly of VLPs during these ITC experiments was confirmed by studying their reaction products using SEC, DLS, and TEM (Figure 5). Figure 5 shows that both with PSS and with ssDNA VLPs are formed in the ITC experiments. For PSS, SEC shows particles eluting at a volume of $V=12 \mathrm{~mL}$, and DLS analysis of these particles (Figure 5b) show they have a diameter of $M_{\mathrm{n}}=17.7 \pm 3.2 \mathrm{~nm}$ (intensity average diameter $\left.\left(M_{\mathrm{i}}\right)=19.5 \pm 3.5 \mathrm{~nm}\right)$, which is in line with the particle size observed in TEM of $18.8 \pm 2.3 \mathrm{~nm}$ (Figure $5 \mathrm{c}$ ). For ssDNA, SEC shows more polydispersity in the particle size, resulting in signals at $V \sim 8$ and $\sim 10 \mathrm{~mL}$. The reason for this is that, due to volumes required for ITC measurements, we used ssDNA derived from salmon testes, which was fragmented by sonication. Over time, this ssDNA can reanneal causing the formation of larger templates for $\mathrm{CP}$ assembly. Isolation of the fraction at $\sim 10 \mathrm{~mL}$ shows particles having an Mn diameter of 


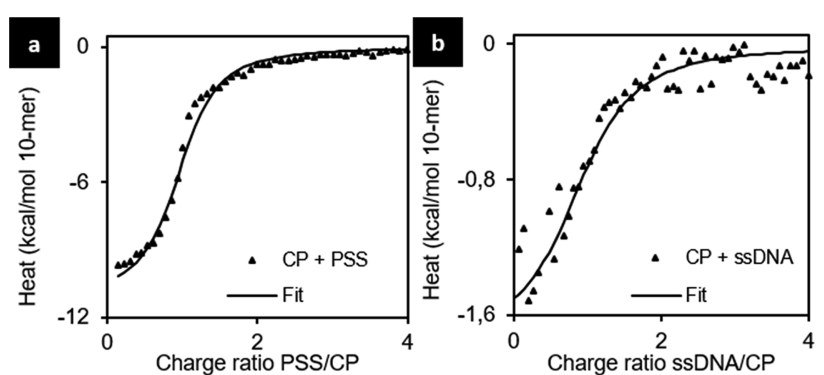

Figure 4. Integrated data of the heat change per injection, normalized to heat per mole of a fraction containing ten charges, of (a) a $6.7 \mathrm{kDa}$ PSS-solution and (b) an ssDNA solution into a CP solution $(\sim 1 \mathrm{mg}$ $\mathrm{mL}^{-1} \mathrm{CP}$ in a $\mathrm{pH} 7.5$ buffer containing $0.3 \mathrm{M} \mathrm{NaCl}$ ). The fits (solid lines) presented in (a) and (b) are based on a single-binding site model.

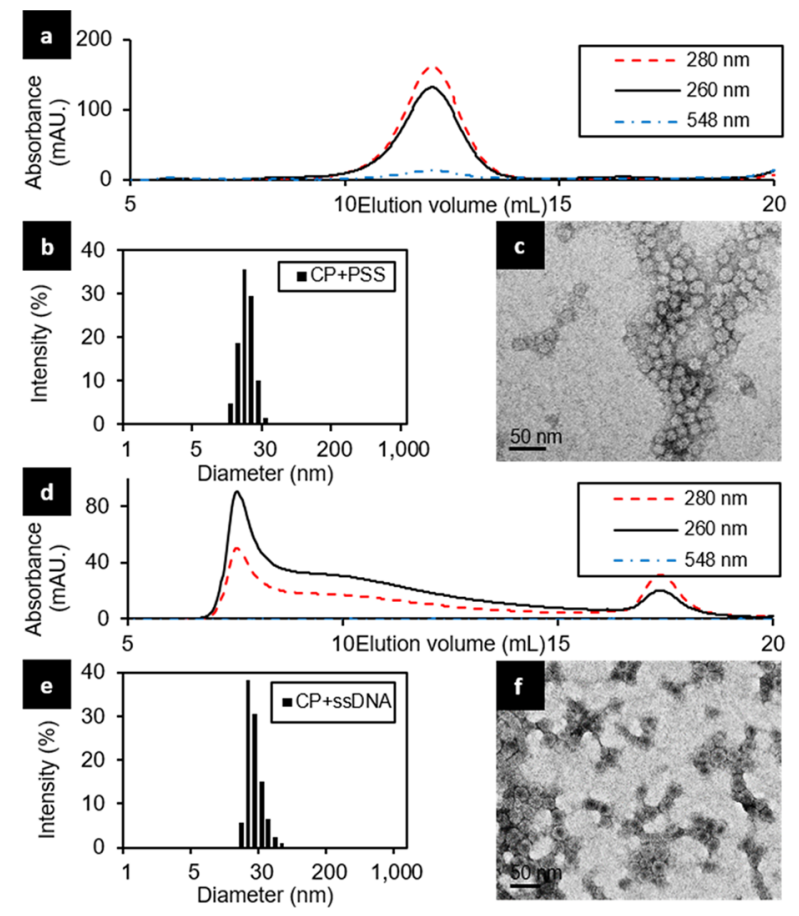

Figure 5. (a) SEC trace, (b) DLS results, and (c) TEM image of the product obtained after ITC where PSS is titrated into a CP solution. (d) SEC trace, (e) DLS results, and (f) TEM image of the product obtained after ITC where ssDNA is titrated into a CP solution $(\sim 1$ $\mathrm{mg} \mathrm{mL}^{-1} \mathrm{CP}$ in a $\mathrm{pH} 7.5$ buffer containing $0.3 \mathrm{M} \mathrm{NaCl}$ ).

$23.8 \pm 4.4 \mathrm{~nm}\left(M_{\mathrm{i}}=60.5 \pm 17.5 \mathrm{~nm}\right.$, indicating the presence of large aggregates) according to DLS measurements and a diameter of $21.9 \pm 2.5 \mathrm{~nm}$ according to TEM. A few larger, rod-like structures were observed in TEM, likely involving reannealed DNA, which were ignored during size measurements. Although affecting the assembly into VLPs, the reannealing of the ssDNA is assumed to have a little effect on the electrostatic interactions between ssDNA and CP, which dominate the ITC measurements.

From Figure 4, we observe that, although both systems show exothermic heat effects, a much larger change in heat is observed upon adding PSS to CP than when ssDNA is added under the same conditions. Note that the PSS $(\sim 6.7 \mathrm{kDa}, \sim 36$ negative charges) and ssDNA ( 500-800 nt, on average 700 negative charges) have a different number of charges per chain. $\mathrm{CP}-\mathrm{PSS}$ and $\mathrm{CP}-$ ssDNA interactions are expected to be primarily of an electrostatic nature; therefore, not the number of molecules but the number of charges added to the CPsolution was kept constant. Similar to the work described in the sections above, where different lengths of PSS are compared, we normalized the integrated ITC data for the number of charges added, giving the heat in kilocalorie per mole of 10-mer segments added.

The ITC data were fitted using the same model used to study CP-PSS interactions (Figure 4) to obtain estimates of the thermodynamic parameters involved in these systems. As before, the 1:1 model that we used does not give an exact representation of the assembly system; however, the inflection point, although steeper in the case of PSS, in both systems occurs at a charge ratio of $\sim 1$ suggesting that charge compensation governs binding in both systems. This observation is in contrast with data published before in which DNA was found to bind with a larger relative number of negative charges (over-compensation). ${ }^{48}$ In this work, it is suggested that initially charge compensation does occur in a random complexation, however upon further capsid assembly, a number of CPs are released leading to over-compensation.

We determined the $K$ of $\mathrm{CP}-\mathrm{PSS}$ interactions to be almost three times higher than of $\mathrm{CP}-$ ssDNA, being $2.8 \times 10^{5}$ and 1.0 $\times 10^{5} \mathrm{M}^{-1}$, respectively. Having $K$ values of the same order of magnitude, no significant differences in affinity are expected between CP and PSS or ssDNA.

Most striking is the differences in $\Delta H$ and $T \Delta S$ observed between these systems. For CP-PSS interactions, $\Delta H$ was determined to be $-11.2 \mathrm{kcal} \mathrm{mol}^{-1}$, being six times higher compared to $\mathrm{CP}-\mathrm{ssDNA}$ yielding $\Delta H=-1.8 \mathrm{kcal} \mathrm{mol}^{-1}$. From $K$ and $\Delta H$, we calculated a $T \Delta S$ of $-3.7 \mathrm{kcal} \mathrm{mol}^{-1}$ for CP-PSS interactions and a $T \Delta S$ of $5.0 \mathrm{kcal} \mathrm{mol}^{-1}$ for CPssDNA interactions. In the experiments discussed earlier, we noticed that $\Delta H$ is primarily affected by the electrostatic CPcargo interactions, which suggests that VLP assembly around PSS is influenced more by the electrostatic interaction. This is tentatively explained by the difference in charge density between PSS and ssDNA, being $\sim 40 \%$ higher for PSS. ${ }^{51}$ Enthalpically, the interaction between CP and PSS seems to be favorable; however entropically, the results show a completely different picture. From $K$ and $\Delta H$, we calculated a $T \Delta S$ of $-3.7 \mathrm{kcal} \mathrm{mol}^{-1}$ for CP-PSS interactions and a $T \Delta S$ of 5.0 $\mathrm{kcal} \mathrm{mol}^{-1}$ for $\mathrm{CP}-\mathrm{ssDNA}$ interactions. However, to obtain more information on this, a further study of the system is required, for example, by making a more extensive comparison between the interactions of the negatively charged groups, either sulfate or phosphate, with the CP. Yet, the data seem to indicate a much more favorable entropy for the DNA-VLP assembly, in line with the presence of hydrophobic interactions and previously reported data. The discrepancy between the PSS and DNA-induced self-assembly may also lie in part in the different particles that are formed, that is, $T=1$ vs $T=3$ particles. Possibly, although creating more and smaller particles, the formation of $T=1$ particles in the case of PSS, with the observed stronger electrostatic interactions, leads to more tightly bound cavities with poorer hydrophobic CP-CP interactions at a higher entropic cost.

We suggest that the large difference in the enthalpic gain, primarily caused by electrostatic $\mathrm{CP}-$ cargo interactions, may be the main explanation for the results obtained when mixing CCMV and PSS where we observe that at $\mathrm{pH} \mathrm{7.5,} \mathrm{when}$ electrostatic interactions stabilize the capsid, the addition of PSS causes CCMV to disassemble and VLPs containing PSS to 
form. We realize that the thermodynamic data presented here are based on a simplified model that cannot yet describe all aspects of the particle assembly accurately and only provides an estimate of the binding affinity between the components involved. Using these estimates and by comparison with nonassembling systems, it does, however, provide substantially improved insight in the assembly parameters and thermodynamic driving forces of the assembly process. Only by fully understanding the assembly pathways, with all its intermediate and final structures, we can accurately determine the strength of all subunit interactions. However, based on our experiments and work described in literature, it seems that weak subunit interactions are a general trend in virus assembly. ${ }^{29,52}$

These weak interactions can be explained by the virus natural infectious pathway. In nature, viruses need to balance between being stable enough to survive various surroundings while still being able to disassemble in their host cells to deliver their viral genome and be able to reproduce. A too strong electrostatic interaction between the $\mathrm{CP}$ and the viral genome would hamper disassembly, which in turn would decrease the virus potential to reproduce. In other studies reported by us, ${ }^{53}$ we have noticed that polyelectrolyte-filled VLPs hardly disassemble, even under very high ionic strength. For virus reproduction, such particle stability would be disadvantageous.

\section{CONCLUSIONS}

We have studied the interactions between CCMV CP and polyanionic cargos PSS and ssDNA. We found that when we mix CCMV and PSS at neutral $\mathrm{pH}, \mathrm{CP}$ is transferred from the virus to PSS to form PSS-filled VLPs. In an attempt to better understand the interactions between $\mathrm{CP}$ and a polyanionic cargo, we investigated the protein cages formed at various PSS to $\mathrm{CP}$ charge ratios using dynamic light scattering and microscale thermophoresis. These measurements suggest that, at all charge ratios, $<1$ particles are being formed, with a maximum number of particles at a charge ratio of $\sim 1$. At a charge ratio $>1$, it seems that no structural changes occur, and the excess PSS is free in solution.

We continued the study of PSS-templated VLP assembly using isothermal titration calorimetry. In line with DLS and MST measurements, binding stoichiometries of $\sim 1$ were found, suggesting that charge compensation governs the assembly process. By separating the electrostatic interactions between $\mathrm{CP}$ and cargo involved in the assembly from the complete assembly, we conclude that $\mathrm{CP}-\mathrm{CP}$ interactions cause an enthalpy change of -3 to $-4 \mathrm{kcal} \mathrm{mol}^{-1}$.

When mixing isolated $\mathrm{CP}$ and PSS or ssDNA at neutral $\mathrm{pH}$ where (mainly electrostatic) cargo-CP interactions drive assembly, significantly higher heat changes were observed when mixing CP with PSS compared to mixing CP with ssDNA. By fitting the ITC data, we estimated that the change in enthalpy caused by the interaction between CP and PSS is approximately six times higher than the change in enthalpy caused by the interaction between $\mathrm{CP}$ and ssDNA. Furthermore, a three times higher $\mathrm{K}$ value was found for the CP-PSS interaction. These parameters likely cause the observed disassembly of CCMV at $\mathrm{pH} 7.5$ and the formation of VLPs containing PSS upon mixing CCMV with PSS when the $\mathrm{CP}-\mathrm{CP}$ interactions are reduced at this increased $\mathrm{pH}$.

The measurements reported here and the tentative interpretation of the data broaden our understanding of the virus assembly in general and specifically adds to our knowledge of the thermodynamics involved. Knowing what the requirements for particle assembly are may aid in the development of new virus-based materials on one hand and on the other could help in the development of new antiviral treatments by understanding what could induce or prevent virus assembly and disassembly. With CCMV as a model system, it can be concluded that fine tuning of the proteincargo interactions can result in the formation of (noninfectious) virus-like particles, although further studies are required, this might be applicable for a variety of viruses with weak protein-protein interactions.

\section{EXPERIMENTAL SECTION}

Materials. All chemicals were purchased from SigmaAldrich and used without further purification unless stated otherwise. PSS (70 kDa) and methacryloxyethyl thiocarbamoyl rhodamine B are purchased from Polysciences. The wild-type CCMV virus is obtained according to literature procedures. $^{54,55}$ Solutions were prepared using Milli-Q water (MQ Millipore, $18.2 \mathrm{~m} \Omega$ ). N-term was purchased from the peptide facility of the Netherlands Cancer Institute.

Wild-Type Capsid Protein Isolation. The CP of CCMV was isolated according to procedures described in the literature. ${ }^{54,55}$ Wild-type CCMV is disassembled by dializing against protein isolation buffer $(50 \mathrm{mM}$ Tris- $\mathrm{HCl}, 500 \mathrm{mM}$ $\mathrm{CaCl}_{2}, 1 \mathrm{mM}$ DTT, pH 7.5) using a 12-14 k MWCO dialysis membrane with 2 times buffer replacement. The high $\mathrm{Ca}^{2+}$ concentration causes the viral RNA to precipitate, and it is removed by $2 \mathrm{~h}$ centrifugation at 40,000 RPM $(179,200 \times g)$ and $4{ }^{\circ} \mathrm{C}$ using a Sorvall WX80 ultracentrifuge. The supernatant containing $\mathrm{CP}$ dimers was dialyzed against a cleaning buffer ( $50 \mathrm{mM}$ Tris- $\mathrm{HCl}, 500 \mathrm{mM} \mathrm{NaCl}, \mathrm{pH} 7.5$ ), followed by dialysis, with 3 times buffer replacement, against capsid storage buffer ( $50 \mathrm{mM} \mathrm{NaOAc}, 1 \mathrm{M} \mathrm{NaCl}, 1 \mathrm{mM}$ $\mathrm{NaN}_{3}, \mathrm{pH} 5$ ), and stored at $4{ }^{\circ} \mathrm{C}$ for a maximum of 2 weeks until further use. To ensure the purity of the protein, only $\mathrm{CP}$ solutions with a $280 / 260 \mathrm{~nm}$ absorbance ratio of at least 1.5 were used.

Rhodamine-Labelled PSS Synthesis (R-PSS). Amounts of $1.0 \mathrm{~g}$ of sodium 4-vinylbenzenesulfonate ( $5 \mathrm{mmol}), 34.2 \mathrm{mg}$ of methacryloxyethyl thiocarbamoyl rhodamine B $(0.05$ $\mathrm{mmol}$ ), and $14.5 \mu \mathrm{L}$ of 2-hydroxyethyl 2-bromoisobutyrate $(20.1 \mathrm{mg}, 0.1 \mathrm{mmol})$ are dissolved in $5 \mathrm{~mL}$ of $\mathrm{MQ}$. This solution is purged with $\mathrm{N}_{2}$ gas for $45 \mathrm{~min}$. An excess amount of methanol is purged with $\mathrm{N}_{2}$ gas for 45 min also. After, $1.7 \mathrm{~mL}$ of purged methanol is added to the aqueous solution using a $\mathrm{N}_{2}$-purged syringe. The solution is purged with $\mathrm{N}_{2}$ gas for an additional 5 min before adding $13 \mathrm{mg}$ of copper(I) bromide $(0.090 \mathrm{mmol})$ and $30 \mathrm{mgof} 2,2^{\prime}$-bipyridyl $(0.19 \mathrm{mmol})$ as a solid while maintaining a $\mathrm{N}_{2}$ gas purge. After addition of the catalyst, the reaction mixture turns brown and is stirred under $\mathrm{N}_{2}$ atmosphere for $24 \mathrm{~h}$ at $21^{\circ} \mathrm{C}$. After $24 \mathrm{~h}$, the reaction is terminated by opening the flask, causing the reaction mixture to turn from brown to blue indicating oxidation of the $\mathrm{Cu}(\mathrm{I})$ catalyst to $\mathrm{Cu}(\mathrm{II})$. The copper is removed by running the mixture over a silica gel column (eluent 1:1 H2O: $\mathrm{MeOH}$ ). After, the polymer was precipitated from THF. The precipitated solid was filtered off, redissolved in 1:1 $\mathrm{H} 2 \mathrm{O}$ : $\mathrm{MeOH}$, and again precipitated from THF. The purified polymer was dried for $12 \mathrm{~h}$ at $60{ }^{\circ} \mathrm{C}$ before analysis using NMR (Figure 6), FPLC, UV-Vis spectroscopy, and fluorescence spectroscopy $\left(\lambda_{\mathrm{ex}}=563 \mathrm{~nm}\right.$ and $\lambda_{\mathrm{em}}=583$ $\mathrm{nm})$. Based on the sodium 4-vinylbenzenesulfonate monomer 


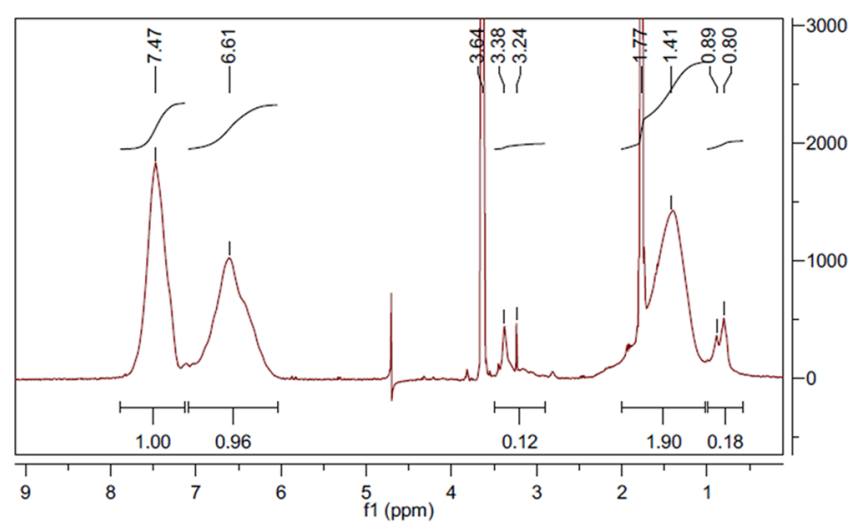

Figure 6. NMR spectrum of R-PSS after purification.

conversion determined by NMR, the molecular weight of the R-PSS is $\sim 6.7 \mathrm{kDa}$.

Size-Exclusion Chromatography. Analysis and purification by size-exclusion chromatography (SEC) were performed using a Superose 6 10/100 GL column on a fast protein liquid chromatography (FPLC) system (GE), eluting with $2 x$ diluted PSS encapsulation buffer (25 mM Tris; $150 \mathrm{mM} \mathrm{NaCl} ; \mathrm{pH}$ 7.5).

Dynamic Light Scattering. The particle size distribution of the VLPs in the various buffers was determined using dynamic light scattering (DLS), using a Microtrac Nanotrac Wave W3043. The viscosity and refractive index of water and the refractive index of native CCMV (1.54) were used in data processing.

UV-vis Spectroscopy. UV-vis spectra were measured in a quartz cuvette using a PerkinElmer Lambda 850 UV-vis spectrometer.

Fluorescence Spectroscopy. Fluorescence excitation and emission spectra were measured in a quartz cuvette using a PerkinElmer LS 55 fluorescence spectrometer.

Transmission Electron Microscopy. For Transmission electron microscopy (TEM), $5 \mu \mathrm{L}$ of sample is drop casted onto a Formvar carbon-coated copper grid. After 1 min of incubation, the remaining liquid is removed using filter paper (Schleicher \& Schuell). The samples are stained using $5 \mu \mathrm{L}$ of a $1 \%$ uranyl acetate in MQ water, which is removed after $20 \mathrm{~s}$ using a filter paper. Samples are imaged using a Philips CM300ST-FEG TEM or a Zeiss Merlin (S)TEM. Particle sizes were determined using ImageJ software.

Nuclear Magnetic Resonance. ${ }^{1} \mathrm{H}$ nuclear magnetic resonance (NMR) spectra were recorded using a Bruker 400 $\mathrm{MHz}$ NMR.

Mass Measurements. Mass spectra were recorded with a Waters electrospray ionization time-of-flight mass spectrometer operated in the positive ion mode (ESI $(+)-\mathrm{ToF}$, Micromass LCT).

Isothermal Titration Calorimetry. Isothermal titration calorimetry (ITC) ITC measurements were carried out using a Microcal VP-ITC with a cell volume of $1.4115 \mathrm{~mL}$. In general, a solution of polyanion in $\mathrm{pH} 7.5$ buffer $(50 \mathrm{mM}$ Tris- $\mathrm{HCl}$, $0.3 \mathrm{M} \mathrm{NaCl}$; unless stated otherwise) was titrated into a solution containing $\mathrm{CP}$ or $\mathrm{N}$-term in the same buffer, while constantly monitoring the heat flux.

\section{ASSOCIATED CONTENT}

\section{S Supporting Information}

The Supporting Information is available free of charge on the ACS Publications website at DOI: 10.1021/acs.jpcb.9b06258.

SEC traces and DLS measurements of CCMV mixed with PSS at $\mathrm{pH} 5$ and $\mathrm{pH}$ 7.5, SDS-PAGE analyses, and TEM image of PSS-containing particles (PDF)

\section{AUTHOR INFORMATION}

\section{Corresponding Author}

*E-mail: J.J.L.M.Cornelissen@utwente.nl.

ORCID

Jurriaan Huskens: 0000-0002-4596-9179

Jeroen J. L. M. Cornelissen: 0000-0002-9728-5043

\section{Author Contributions}

The manuscript was written through contributions of all authors.

\section{Funding}

European research council - CoG Protcage [616907].

Notes

The authors declare no competing financial interest.

\section{REFERENCES}

(1) Krishna, O. D.; Kiick, K. L. Protein- and peptide-modified synthetic polymeric biomaterials. Biopolymers 2010, 94, 32-48.

(2) Niemeyer, C. M. Functional Hybrid Devices of Proteins and Inorganic Nanoparticles. Angew. Chem., Int. Ed. 2003, 42, 5796-5800.

(3) Obermeyer, A. C.; Olsen, B. D. Synthesis and application of protein-containing block copolymers. ACS Macro Lett. 2015, 4, 101110.

(4) Lee, S. Y.; Lim, J. S.; Harris, M. T. Synthesis and application of virus-based hybrid nanomaterials. Biotechnol. Bioeng. 2012, 109, 1630.

(5) Soto, C. M.; Ratna, B. R. Virus hybrids as nanomaterials for biotechnology. Curr. Opin. Biotechnol. 2010, 21, 426-438.

(6) Ludwig, C.; Wagner, R. Virus-like particles-universal molecular toolboxes. Curr. Opin. Biotechnol. 2007, 18, 537-545.

(7) Pokorski, J. K.; Steinmetz, N. F. The art of engineering viral nanoparticles. Mol. Pharmaceutics 2010, 8, 29-43.

(8) Wang, Q.; et al. Icosahedral virus particles as addressable nanoscale building blocks. Angew. Chem., Int. Ed. 2002, 41, 459-462.

(9) Lee, Y. J.; et al. Fabricating genetically engineered high-power lithium-ion batteries using multiple virus genes. Science 2009, 324, $1051-1055$.

(10) Nam, K. T.; et al. Virus-enabled synthesis and assembly of nanowires for lithium ion battery electrodes. Science 2006, 312, 885888.

(11) Czapar, A. E.; Steinmetz, N. F. Plant viruses and bacteriophages for drug delivery in medicine and biotechnology. Curr. Opin. Chem. Biol. 2017, 38, 108-116.

(12) Garcea, R. L.; Gissmann, L. Virus-like particles as vaccines and vessels for the delivery of small molecules. Curr. Opin. Biotechnol. 2004, 15, 513-517.

(13) Malyutin, A. G.; et al. Viruslike nanoparticles with maghemite cores allow for enhanced mri contrast agents. Chem. Mater. 2014, 27, 327-335.

(14) Huang, X.; et al. Self-Assembled Virus-like Particles with Magnetic Cores. Nano Lett. 2007, 7, 2407-2416.

(15) Bode, S. A.; et al. Reactions inside nanoscale protein cages. Nanoscale 2011, 3, 2376-2389.

(16) Liu, A.; Traulsen, C. H. H.; Cornelissen, J. J. L. M. Nitroarene Reduction by a Virus Protein Cage Based Nanoreactor. ACS Catal. 2016, 6, 3084-3091.

(17) Minten, I. J.; et al. Catalytic capsids: The art of confinement. Chem. Sci. 2011, 2, 358-362. 
(18) Patterson, D. P.; Prevelige, P. E.; Douglas, T. Nanoreactors by Programmed Enzyme Encapsulation Inside the Capsid of the Bacteriophage P22. ACS Nano 2012, 6, 5000-5009.

(19) Bancroft, J. B.; Hills, G. J.; Markham, R. A study of the selfassembly process in a small spherical virus formation of organized structures from protein subunits in vitro. Virology 1967, 31, 354-379.

(20) Lavelle, L.; et al. Phase diagram of self-assembled viral capsid protein polymorphs. J. Phys. Chem. B 2009, 113, 3813-3819.

(21) Endres, D.; Zlotnick, A. Model-based analysis of assembly kinetics for virus capsids or other spherical polymers. Biophys. J. 2002, $83,1217-1230$

(22) Hagan, M. F.; Elrad, O. M.; Jack, R. L. Mechanisms of kinetic trapping in self-assembly and phase transformation. J. Chem. Phys. 2011, 135, 104115.

(23) van der Schoot, P.; Zandi, R. Kinetic theory of virus capsid assembly. Phys. Biol. 2007, 4, 296-304.

(24) Zlotnick, A.; et al. A Theoretical Model Successfully Identifies Features of Hepatitis B Virus Capsid Assembly. Biochemistry 1999, 38, 14644-14652.

(25) Zlotnick, A.; Porterfield, J. Z.; Wang, J. C.-Y. To Build a Virus on a Nucleic Acid Substrate. Biophys. J. 2013, 104, 1595-1604.

(26) Hagan, M. F. A theory for viral capsid assembly around electrostatic cores. J. Chem. Phys. 2009, 130, 114902.

(27) Tars, K. Introduction to Capsid Architecture. In Viral Nanotechnology; Khudyakov, Y.; Pumpens, P. Editor; 2015, CRC Press: 524.

(28) Crick, F. H. C.; Watson, J. D. Structure of small viruses. Nature 1956, 177, 473-475.

(29) Perlmutter, J. D.; Perkett, M. R.; Hagan, M. F. Pathways for Virus Assembly around Nucleic Acids. J. Mol. Biol. 2014, 426, 31483165.

(30) Nebel, S.; Bartoldus, I.; Stegmann, T. Calorimetric detection of influenza virus induced membrane fusion. Biochemistry 1995, 34, $5705-5711$.

(31) Jeembaeva, M.; et al. DNA Heats Up: Energetics of Genome Ejection from Phage Revealed by Isothermal Titration Calorimetry. J. Mol. Biol. 2010, 395, 1079-1087.

(32) Julien, J. P.; et al. Asymmetric recognition of the HIV-1 trimer by broadly neutralizing antibody PG9. Proc. Natl. Acad. Sci. U. S. A. 2013, 110, 4351-4356.

(33) King, N. M.; et al. Structural and thermodynamic basis for the binding of TMC114, a next-generation human immunodeficiency virus type 1 protease inhibitor. J. Virol. 2004, 78, 12012-12021.

(34) de Souza, T. L. F.; et al. Charge neutralization as the major factor for the assembly of nucleocapsid-like particles from C-terminal truncated hepatitis C virus core protein. PeerJ 2016, 4, e2670.

(35) Maassen, S. J.; et al. Oligonucleotide Length-Dependent Formation of Virus-Like Particles. Chem. - Eur. J. 2018, 24, 74567463.

(36) Caspar, D. L. D. Assembly and Stability of the Tobacco Mosaic Virus Particle. In Advances in Protein Chemistry; Anfinsen, C. B.; Anson, M. L.; Edsall, J. T. Editors; 1964, Academic Press.: 37-121.

(37) Liu, H.; et al. Pseudo-atomic models of swollen CCMV from cryo-electron microscopy data. J. Struct. Biol. 2003, 142, 356-363.

(38) Speir, J. A.; et al. Structures of the native and swollen forms of cowpea chlorotic mottle virus determined by X-ray crystallography and cryo-electron microscopy. Structure 1995, 3, 63-78.

(39) Singh, S.; Zlotnick, A. Observed hysteresis of virus capsid disassembly is implicit in kinetic models of assembly. J. Biol. Chem. 2003, 278, 18249-18255.

(40) Caspar, D. L. D.; Klug, A. Physical principles in the construction of regular viruses. In Cold Spring Harbor symposia on quantitative biology; Cold Spring Harbor Laboratory Press: 1962, 27, $1-24$.

(41) Garmann, R. F.; et al. Role of electrostatics in the assembly pathway of a single-stranded RNA virus. J. Virol. 2014, 88, 1047210479.

(42) Note: The slight deviation of the values for CP-PSS interactions described here, compared to those shown above are likely due to the use of a different batch of $\mathrm{CP}$ or experimental and data fitting errors.

(43) Johnson, J. M.; et al. Regulating self-assembly of spherical oligomers. Nano Lett. 2005, 5, 765-770.

(44) Ceres, P.; Zlotnick, A. Weak Protein-Protein Interactions Are Sufficient To Drive Assembly of Hepatitis B Virus Capsids. Biochemistry 2002, 41, 11525-11531.

(45) Prevelige, P. E., Jr.; King, J.; Silva, J. L. Pressure denaturation of the bacteriophage P22 coat protein and its entropic stabilization in icosahedral shells. Biophys. J. 1994, 66, 1631-1641.

(46) Maassen, S. J.; van der Schoot, P.; Cornelissen, J. J. L. M. Experimental and Theoretical Determination of the $\mathrm{pH}$ inside the Confinement of a Virus-Like Particle. Small 2018, 14, 1802081.

(47) Melander, W.; Horváth, C. Salt effects on hydrophobic interactions in precipitation and chromatography of proteins: An interpretation of the lyotropic series. Arch. Biochem. Biophys. 1977, $183,200-215$.

(48) Cadena-Nava, R. D.; et al. Self-assembly of viral capsid protein and RNA molecules of different sizes: Requirement for a specific high protein/RNA mass: Ratio. J. Virol. 2012, 86, 3318-3326.

(49) Hu, Y.; et al. Packaging of a Polymer by a Viral Capsid: The Interplay between Polymer Length and Capsid Size. Biophys. J. 2008, 94, 1428-1436.

(50) Cadena-Nava, R. D.; et al. Exploiting Fluorescent Polymers To Probe the Self-Assembly of Virus-like Particles. J. Phys. Chem. B 2011, $115,2386-2391$.

(51) Hernandez-Garcia, A.; Cohen Stuart, M. A.; De Vries, R. Templated co-assembly into nanorods of polyanions and artificial virus capsid proteins. Soft Matter 2017, 14, 132-139.

(52) Zlotnick, A. Are weak protein-protein interactions the general rule in capsid assembly? Virology 2003, 315, 269-274.

(53) Brasch, M.; et al. Encapsulation of phthalocyanine supramolecular stacks into virus-like particles. J. Am. Chem. Soc. 2011, 133, $6878-6881$.

(54) Verduin, B. J. M. The preparation of CCMV-protein in connection with its association into a spherical particle. FEBS Lett. 1974, 45, 50-54.

(55) Comellas-Aragonès, M.; et al. A virus-based single-enzyme nanoreactor. Nat. Nanotechnol. 2007, 2, 635-639. 